\title{
Electron transfer and transport through multi-heme proteins: recent progress and future directions
}

\author{
Jochen Blumberger
}

June 20, 2018

University College London, Department of Physics and Astronomy, Gower Street, London WC1E 6BT, UK.

\begin{abstract}
I review recent experimental measurements probing electron transfer (ET) and electron transport (ETp) through multi-heme cytochromes (MHCs) as well as their theoretical interpretation. Examples include pump-probe spectroscopy of Ru-labeled MHCs aimed at determining heme-heme ET rates in MHCs and the measurement of the $I-V$ characteristics of MHCs in bioelectronic junctions. While the ET mechanism appears to be well established for MHCs in aqueous solution, the ETp mechanism in bioelectronic junctions such as STM remains elusive partly due to the complexities of the electrode-protein interface.
\end{abstract}

to whom correspondence should be sent, e-mail: j.blumberger@ucl.ac.uk 


\section{Introduction}

The bacterium $S$. Oneidensis has evolved one of the most astonishing survival mechanisms in response to low oxygen concentrations. ${ }^{1}$ When cytoplasmatic $\mathrm{O}_{2}$ becomes scarce it starts to grow $\mu$ m-long electrically conducting cellular appendages, which allows the bacterium to export electrons from the cytoplasm to extracellular space for reduction of extracellular electron acceptors in place of $\mathrm{O}_{2}$ (see Figure 1A). ${ }^{2-4}$ Until not too long ago it was speculated that these conducting appendages are bacterial pili, believed to be relevant for extracellular electron transport in other organisms, e.g. G. sulfurreducens. ${ }^{5-7}$ However, recent studies on $S$. Oneidensis demonstrated that the rigid fibers protruding from the cell surface are in fact extensions of the outer membrane and the periplasm into tubular vesicles. ${ }^{3,4,8}$ Their electric conductivity, which rivals the one of man-made organic semiconductors, ${ }^{9}$ is thought to be conferred to these structures by multi-heme cytochromes $(\mathrm{MHC})^{10-13}$ that form wire-like complexes spanning the outer membrane (Figure 1B-D). ${ }^{3,14,15}$

MHCs have attracted much interest for some time, as they are thought to facilitate electron transport in mediatorless microbial fuel cells, ${ }^{16,17}$ in microbial electrosynthesis, ${ }^{18-20}$ in the decontamination of water and soil containing radioactive isotopes ${ }^{21}$ and in bionanotechnological applications. ${ }^{9}$ However, many fundamental properties of these fascinating biomolecules are still not well understood. What is the magnitude of the intrinsic electron flow that these biomolecules support? What parts of the protein limit the electron flow? How does conductivity change when moving from an aqueous to a non-polar environment or to air? What is the mechanism of conduction? Can we design mutations that allow us to control magnitude and directionality of electron flow? None of these questions can be currently answered with certainty, although efforts by several groups have been undertaken in recent years that have given us first insights and useful clues.

Here I start with a brief review of recently resolved structures of MHCs. Then I place focus on experimental measurements of electronic properties of single $\mathrm{MHCs}^{22-24}$ and their complexes (nm length scale) ${ }^{15}$ as well as their interpretation by theory and computation. ${ }^{24-31}$ In this respect I distinguish between electron transfer (ET) and electron transport (ETp) measurements. In ET an electron transfers between an electron donor and an electron acceptor resulting in a change of their net charge, which is accompanied by dielectric relaxation of the environment if present. ${ }^{32}$ This process is usually characterized by a chemical (here ET) rate constant. In biology it is often the case that several successive ET events occur along a chain of redox cofactors with each ET event characterized by a rate constant. An example that will be discussed here is electron injection in MHCs via molecular donor groups and the subsequent ET through the protein. ${ }^{15,22}$ On the other hand, ETp is defined as the flow of electrons through a molecule (or biomaterial) without a change of the net charge of that molecule or donor/acceptor groups (e.g. redox cofactors) within that molecule. ${ }^{32}$ In practice, ETp is realised by application of a bias voltage to the termini of the molecule and the electric current replaces the ET rate constant as the experimental observable. In this review we will discuss ETp through MHCs that are sandwiched between a metal substrate and an STM tip. ${ }^{23,24,33}$

A more comprehensive review on ET in MHCs covering developments until 2014 can be found in Ref. 34 and a review on protein ETp and bioelectronics has been published very recently. ${ }^{32}$ For ETp measurements on cellular length scales and beyond, e.g. on appendages of S. Oneidensis ${ }^{35}$ and in biofilms of $G$. sulfurreducens, ${ }^{5-7,36-38}$ and for modelling work thereof, ${ }^{39,40}$ I refer to the recent literature.

\section{Structures of MHCs and cellular appendages}

High resolution structures have been resolved for numerous MHCs from the organism S. oneidensis MR-1, binding 4 hemes (quinol oxidase Cym A, ${ }^{41}$ small tetraheme cytochrome STC, ${ }^{42}$ fumerate reductase FccA ${ }^{43}$ ), 5 and 8 hemes (oxoanion reductases $\mathrm{NrfA}^{44}$ and $\mathrm{OTR}^{45}$ ), 10 hemes (outer-membrane cytochromes $\mathrm{MtrF}^{46}$ and $\mathrm{MtrC}^{47}$ ) and 11 hemes (UndA). ${ }^{11}$ While some of these proteins catalyze redox chemistry (Cym A, FccA, NrfA, OTR), others are thought to function as electron storage (STC) and electron transport proteins (MtrC, $\mathrm{MtrF})$. The first crystal structure of a deca-heme protein of was resolved in 2011 (MtrF), ${ }^{46}$ though with relatively low resolution. The most recent addition came in 2015 with the structure of $\mathrm{MtrC},{ }^{47}$ obtained at much improved resolution, see Figure 1C. 
The MHC crystal structures reveal close-packed, chain-like, bis-His coordinated Fe-heme arrangements on the 1-10 nm scale, which is why they are sometimes referred to as "nanowires". Each heme contains iron coordinated by protoporphyrin IX (c-heme) that is covalently linked to the peptide by two thioether bonds arising from a Cys-X1-X2-Cys-His heme-binding motif that provides a His axial ligand to the heme iron and where $\mathrm{X}$ can be any amino acid. In most structures a second axial His ligand is provided by the peptide chain. The bis-His coordination results in a low-spin configuration of $\mathrm{Fe}$ in reduced $\left(\mathrm{Fe}^{2+}\right)$ and oxidized states $\left(\mathrm{Fe}^{3+}\right)$. Despite the different protein folds and molecular weights of these proteins, the hemes arrange in remarkably few motifs that differ in their heme edge-to-edge distances and hence the ET rate constants they support, the latter usually decreasing from "stacked" (Figure 1D) to "T-shaped" to "coplanar" motif. ${ }^{27}$

Electron transport from the cytoplasm to extracellular space requires a sophisticated transport system involving multiple soluble MHCs in the periplasm as well as protein complexes made of MHCs that transport electrons across the outer membrane. Examples for the latter are the MHC protein complexes MtrCAB and MtrFDE of S. Oneidensis ${ }^{14}$ as well as the recently identified OmaX/OmbX/OmcX (X=B,C) protein complexes of G. sulfurreducens. ${ }^{48}$ Extended electron transport away from the cell surface is facilitated by bacterial appendages formed at reduced $\mathrm{O}_{2}$ levels as mentioned above. Much progress has been made very recently to obtain insight into the nature of these fascinating structures. ${ }^{3,8}$ Using correlative light microscopy and electron cryotomography, they were identified as chains of interconnected outer-membrane vesicles with periplasmatic and outer-membrane cytochromes MtrA and MtrC, respectively, distributed along their length ${ }^{8}$ (schematically shown in Figure $1 \mathrm{~B}^{3}$ ). Based on the observed cytochrome packing density, a plausible ET path along the OM extensions involving a combination of direct hopping and cytochrome diffusion was proposed. ${ }^{8}$

\section{Electron transfer kinetics}

Proteoliposome experiment. One of the first experiments probing ET kinetics through MHCs were carried out on the 20-heme complex MtrCAB, comprised of two deca-heme proteins MtrC and MtrA and the structural non-heme protein MtrB. ${ }^{15}$ The complex was successfully inserted in a proteoliposome mimicking the bacterial membrane, with MtrC outward facing and in contact with an Fe(III)-oxide $\left(\mathrm{Fe}_{2} \mathrm{O}_{3}\right)$ nanoparticle, see Figure 2A. Electron supply was provided inside the proteoliposome by excess methyl viologen (MV). It was shown that the MtrCAB complex facilitated ET from MV to the Fe(III)-nanoparticle resulting in the near-quantitative formation of aqueous $\mathrm{Fe}$ (II) at a rate of about $10,000 \mathrm{~s}^{-1}$, see Figure $2 \mathrm{~B}$. The reported rate was dependent on the type of iron oxide mineral used implying that electron flow was limited by interfacial $\mathrm{ET}$ at the MtrC/mineral interface. Hence, the observed rate should be regarded as a lower bound to the intrinsic electron flow supported by MtrCAB.

Pump-probe spectroscopy. One of the most important properties that have eluded experimental measurements so far are the heme-to-heme ET rate constants in single MHCs that govern the intrinsic protein-limited electron flow through these structures. Their determination is difficult in general because of the identical chemical nature of the heme groups. A promising approach for probing ET kinetics is pumpprobe spectroscopy on MHCs labeled with covalently linked $\mathrm{Ru}(\mathrm{II})-\mathrm{L}$ chromophores, where $\mathrm{L}$ is a bipyridine derivative. $^{22}$ The idea is that upon light absorption a metal-to-ligand charge transfer excited state $\mathrm{Ru}(\mathrm{III}) \mathrm{L}^{-}$ is formed that has sufficient energy to inject an electron to a nearby heme cofactor of the protein (Figure 2D). Subsequent ET along the heme chain, if it occurs, could be followed spectroscopically. In Ref. 22 a $\mathrm{Ru}$-label was positioned at different locations on the protein surface of the tri-heme cytochrome $\mathrm{c}_{7}$ from Geobacter sulfurreducens (PpcA), ${ }^{22}$ see Figure $2 \mathrm{C}$. The excited state decay times were found to vary from 6 ps to $40 \mathrm{~ns}$ correlating well with the distance and pathways for electron injection into the nearest heme. However, clear evidence for subsequent inter-heme electron transfer within PpcA was not detected on this time scale. I note that the absence of heme-heme ET on this time scale is in line with latest computational predictions for the electron flux across a similar protein from S. Oneidensis, STC, estimated to be in the order of $10^{7} \mathrm{~s}^{-1} \cdot{ }^{29}$ Hence, an extension of the measurements of Ref. 22 to the microsecond regime would be instructive to verify some of the predictions of recent computations. 
Theory and Computation. Arguably, most of what we think we know about the molecular details of ET thermodynamics and kinetics in MHC is based on predictions from molecular simulations and electronic structure calculations. In an early study, Breuer et al. reported redox potentials for all 10 hemes in alloxidized MtrF using MD simulation, ${ }^{25}$ and very recently Barrozo et al. reported heme redox potentials for MtrF and MtrC in all-ox and all-red states. ${ }^{31}$ Both studies agreed that the free energy profile for electron flow along the wire has ups and downs, yet resulting in near thermoneutral ET along the long axis of the protein (heme 5 to 10). In terms of kinetics, several ET mechanisms have been investigated including bandlike, ${ }^{49}$ flickering resonance (FR), ${ }^{28,50}$ superexchange $(\mathrm{SE})^{28}$ and electron hopping. ${ }^{24,27,28,49,51}$ Our group concluded that ET across the solvated protein occurs via stepwise (incoherent, non-adiabatic) electron hopping between neighbouring $\mathrm{Fe}^{2+} / \mathrm{Fe}^{3+}$ heme pairs, ${ }^{28}$ in agreement with previous suggestions. ${ }^{49,51}$ The reason is that electronic coupling between the heme cofactors, at most a few meV, ${ }^{27,29}$ is too small for band theory to apply ${ }^{49}$ and not sufficiently large to make the FR or SE ET mechanisms competitive with hopping. ${ }^{28}$ We note in passing that the FR mechanism has been recently suggested for DNA base pairs where electronic couplings can be significantly larger than in MHCs. ${ }^{50}$ Moreover, band-like transport mediated by pi-stacked amino acids was proposed in nanowire (conductive type IV pili) containing biofilms of $G$. sulfurreducens. ${ }^{5,38}$ Yet the mechanism of pili conduction, band-like vs redox hopping as inferred from the temperature dependence of the electronic conductivity, is still a matter of debate ${ }^{37}$ and was recently shown to depend on environmental conditions (life biofilms vs purified pili). ${ }^{7}$

Returning to ET in MHCs, Breuer et al. calculated all ET (Marcus) parameters (electronic coupling matrix elements and reorganization free energies in addition to redox potentials) and rate constants for heme-heme ET in MtrF. ${ }^{27}$ Using these rates to solve a Master equation for electron hopping along the MtrF wire, he estimated the maximum, protein-limited electron flux for this protein to be in the order of $10^{4}-10^{5} \mathrm{~s}^{-1}$. Somewhat surprisingly, this is just slightly above the electron flow through MtrCAB determined in the (interfacial ET-limited) proteoliposome experiments. ${ }^{15}$ Recent calculations on the tetraheme cytochrome STC suggested that cysteine linkages, which covalently attach the hemes to the protein frame, significantly increase heme-heme electronic couplings resulting in electron flux enhancements by a factor of 37 for aqueous STC. ${ }^{29}$ The cysteine linkages were not included in the coupling calculations for MtrF suggesting that the protein-limited electron flux through MtrF could be 1-2 orders of magnitude higher than previously reported, ${ }^{27}$ possibly exceeding $10^{6} \mathrm{~s}^{-1}$.

\section{Electron transport in bioelectronic junctions}

Complementary to solution phase ET kinetics, ETp through MHCs was probed by scanning tunneling microscopy (STM) in air or vacuum, see Figure 3A. In one of the first studies of this kind, MtrC and the related deca-heme protein OmcA could be immobilized on a flat $\mathrm{Au}$ substrate and the current response measured as a function of bias applied between STM tip and substrate. ${ }^{23}$ It was found that both proteins support nA tunneling currents at modest bias voltages, but they featured different $I-V$ profiles (Figure 3B). The current response for OmcA was symmetric and could be well described by a coherent elastic tunneling model $(\mathrm{CET})^{52,53}$ suggesting that the charge carriers do not localize on Fe-heme electronic states during transport. ${ }^{30}$ This does not exclude the possibility, however, that Fe-heme electronic states are involved in the conduction process. On the contrary, one would expect that the highest occupied and the lowest unoccupied electronic energy levels of the MHCs are composed of Fe $\mathrm{d}\left(t_{2 g}\right)$-heme and Fe $\mathrm{d}\left(e_{g}\right)$-heme orbitals, respectively, that linearly combine to form extended states delocalized over the whole heme chain. One may speculate that these states mediate the elastic tunneling current, resonantly or off-resonantly depending on how they align with respect to the Fermi-levels of the electrodes. Future electronic structure calculations should bring some more clarity on this mechanistic aspect.

Intriguingly, the $I-V$ profile for MtrC differed from the one for OmcA featuring significant breaks in slope at positive tip bias. The increased conductance in these regions were attributed to mediation of the tunneling current by localized heme electronic states suggesting that charge carriers localize at least transiently on heme groups. ${ }^{30}$ More recent STM measurements on MtrF single proteins also reported nA 
tunneling current ${ }^{24}$ (Figure 3C), yet without the tunneling enhancements reported previously for MtrC. One should note however, that such fine details may be obscured by the relatively large error bars of singlemolecule STM measurements. As noted in a recent summary of literature data, STM measurements have a wide spread, which can be due to factors such as the presence of vacuum or air (as in Ref. 24,30), gap contact geometry, and/or a relatively small signal to noise ratio. ${ }^{33}$

Are MHCs better semi-conductors than proteins containing only one or no redox active cofactors? Although I am unaware of a systematic comparison for the same experimental set-up and conditions, I believe the answer to this question is yes, quite likely. The effective tunneling barrier for the deca-heme protein OmcA, obtained from a fit of the STM data to the Simmons equation for coherent elastic tunneling 52,53 is $\phi=1.75 \mathrm{eV}^{30}$ translating into an exponential tunneling decay factor $\beta=1.025 \sqrt{\phi}=1.35 \AA^{-1}(\phi$ in eV). A preliminary analysis of $I-V$ data for immobilized, dry monolayers of azurin, ${ }^{54,55}$ cytochrome $c^{56}$ and a poly-alanine peptide chain ${ }^{57}$ gives higher effective tunneling barriers and decay factors. This comparison should be considered with caution, however, as STM and protein mono-layer junctions are two different techniques and there is evidence that measured currents are very sensitive to the details of the electrode/protein interface (physisorbed vs chemisorbed, nature of linker, orientation of protein on surface etc.) ${ }^{32} I-V$ measurements for dry MHC mono-layer junctions are currently underway - they will permit a comparison with the above mentioned proteins and peptides on a more equal footing.

\section{Conclusions and Perspectives}

We have discussed three types of experiments, pump-probe spectroscopy and proteoliposome studies, both probing ET, and STM measurements probing ETp through MHCs. These techniques differ in several important aspects, e.g. the way charge is injected in the MHC (excited state vs thermal vs interfacial ET), the environmental conditions (aqueous vs lipid bilayer vs air/vacuum) and supply of external electrons (stoichiometric vs diffusion limited vs unlimited). This makes a direct comparison of the results difficult and it should not be surprising if they lead to different mechanistic proposals, especially when comparing ET from kinetic measurements in solution with ETp from current measurements in air/vacuum. The liposome experiment is arguably the most relevant for unperturbed, in vivo situations, whereas the STM and protein mono-layer junction experiments discussed are relevant for nanobioelectronic applications including manipulation of electron flow in and out of bacterial cells.

Theory and computation have given us important clues with regard to the thermodynamics and kinetics of electron transfer through fully solvated MHCs. All computational evidence points to heme-heme electron hopping in the non-adiabatic (Marcus) regime as the transport mechanism in aqueous solution. In future, ET parameters should be refined for MtrF and MtrC in aqueous solution and calculated for conditions that are more akin to the native environment of these proteins. Redox potentials and reorganization free energies are expected to be sensitive to the different dielectric environment in the outer-membrane compared to aqueous solution. These efforts may help us understand observed functional differences between the two proteins. ${ }^{31}$

As always, computational predictions are subject to uncertainties (e.g. protonation state of the protein) and inaccuracies (e.g. protein force field and density functional theory calculations), and await experimental verification. Arguably, spectroscopic pump-probe experiments on chromophore-labeled MHCs are the most promising approach to obtain robust experimental estimates for MHC heme-heme ET rates in future. However, this approach faces two (possibly more) challenges that need to be overcome (i) The hemes are difficult to distinguish with UV/VIS or EPR as they are all bis-His coordinated and low spin. Creation of spectroscopically unique "reporter"-hemes through suitable chemical modification could be a viable way to track electron transfer along the heme wire. (ii) Electron recombination and regeneration of $\mathrm{Ru}(\mathrm{II})$ is likely to be very fast competing with ET along the heme chain. Use of less oxidizing chromophores, e.g. through suitable choice of electron donating bipyridine substituents, could be a remedy.

Their high electronic conductivities in STM junctions make MHCs promising candidates for future nanobioelectronic applications. There is some evidence that the current detected in these measurements is 
limited by the interfacial ET step from the electrode to the protein obscuring the intrinsic, protein-limited current, which may well be even higher. The mechanism for ETp in STM measurements is less clear than for ET in solution. Especially the role of the hemes and the influence of the electrode-protein interface in the conduction process are still not well understood. There is some evidence that MHCs conduct better than proteins of similar size containing only one or no redox active cofactors, which could point to the involvement of the hemes in the conduction process. Future $I-V$ measurements in mono-layer junction configurations on native MHCs, site-specific MHC mutants and for different electrode materials could give further important clues on the conduction mechanism.

To make further progress with theoretical modelling of $I-V$ measurements one needs to obtain (i) a reliable structural model of the MHCs adsorbed on the electrode (ii) sufficiently accurate estimates for the heme redox levels (and other protein level) alignment relative to the Fermi-levels of the electrodes, and (iii) accurate and effective ways to incorporate partial nuclear relaxation (or "partially elastic" ET) in the model as the time for electron transit between the two electrodes (ps-ns) is on the same time scale as typical protein relaxation processes. I am hopeful we will better understand the conduction mechanism in these fascinating biological structures in the years to come through fruitful interplay between theory and experiment.

\section{Acknowledgments}

I would like to thank Julea Butt and David Cahen for stimulating discussions on this topic and acknowledge financial support from EPSRC, Grant No. EP/M001946/1, and from the European Research Council (ERC) under the European Unions Horizon 2020 research and innovation programme, grant agreement no. 682539/SOFTCHARGE.

\section{Recommended Reading}

Papers of particular interest, published within the period of review, have been highlighted as:

- of special interest

$\bullet$ of outstanding interest

- Pirbadian (2014):

The first in-vivo observations of formation of bacterial appendages ("nanowires") in S. Oneidensis are reported suggesting that they are extensions of the outer-membrane and periplasm rather than pilin-based structures.

•• Subramanian (2018):

Follow-up work from Pirbadian (2014), showing high-resolution images of S. Oneidensis outer membrane extensions confirming they are chains of interconnected outer membrane vesicles with variable extent of tubulation.

- Leung (2013):

A field-effect transistor (FET) is constructed from individual nanowires from $S$. Oneidensis showing that they exhibit p-type electronic behaviour with field-effect mobilities of $0.1 \mathrm{~cm}^{2} \mathrm{~V}^{-1} \mathrm{~s}^{-1}$, comparable to devices based on synthetic organic semiconductors.

$\bullet$ White (2013):

The MtrCAB 20-heme complex is successfully inserted in a proteoliposome and shown to facilitate electron flow from the inside to the outside of the proteoliposome on to an iron-oxide nanoparticle.

$\bullet$ Kokhan (2015):

Pump-probe experiments on Ru-labeled tri-heme cytochrome PpcA showing evidence for photo-excited electron transfer from the Ru-label to the protein. Such experiments could give us important information on heme-heme ET rate constants.

$\bullet$ Byun (2014): 
STM measurements are reported for MtrF at low and high humidity conditions. The $I-V$ profiles showing nano-Ampere currents are fit to a hopping model for electronic conduction.

- Breuer (2012):

First report of computed redox potentials for MtrF from molecular dynamics simulation and of the corresponding free energy landscape for electron transfer along this deca-heme protein.

•• Breuer (2014):

First report of all Marcus parameters, heme-heme ET rate constants and protein-limited electron flow for MtrF suggesting that thermodynamically up-hill steps occur between electronically well connected heme pairs.

- Blumberger (2015):

A comprehensive review on recent theoretical advances in the field of biological electron transfer, covering developments until 2015.

- Jiang (2017):

A DFT study suggesting that cysteine-linkages significantly enhance electronic coupling for the terminal heme pairs in the tetraheme protein STC, thereby enhancing the electron flow through the protein by 1-2 orders of magnitude.

•• Wigginton J. Phys. Chem. B (2007):

One of the first STM measurements of the $I-V$ characteristic of multi-heme proteins, showing that MtrC and OmcA support nano-Ampere currents at modes bias voltages. This paper investigates possible mechanisms for electron conduction in these proteins.

- Bostick (2018):

An authoritative review on the current state of the art of dry protein-monolayer junction measurements.

•• Breuer (2015):

A focused review on multi-heme cytochromes of $S$. oneidensis covering protein structures, protein-film voltammetry measurements, computation of ET thermodynamics and kinetics in MHCs and STM measurements of electron conduction, until 2014.

\section{References}

[1] C. R. Myers, K. H. Nealson, Bacterial manganese reduction and growth with manganese oxide as the sole electron acceptor, Science 240 (1988) 1319-1321.

[2] Y. Gorby, S. Yanina, J. McLean, K. Rosso, D. Moyles, A. Dohnalkova, T. Beveridge, I. Chang, B. Kim, K. Kim, D. Culley, S. Reed, M. Romine, D. Saffarini, E. Hill, L. Shi, D. Elias, D. Kennedy, G. Pinchuk, K. Watanabe, S. Ishii, B. Logan, K. H. Nealson, J. K. Fredrickson, Electrically conductive bacterial nanowires produced by shewanella oneidensis strain mr-1 and other microorganisms, Proc. Nat. Acad. Sci. USA 103 (2006) 11358.

[3] S. Pirbadian, S. E. Barchinger, K. M. Leung, H. S. B. Jangir, R. A. Bouhenni, S. B. Reed, M. F. Romine, D. A. Saffarini, L. Shi, Y. A. Gorby, J. H. Golbeck, M. Y. El-Naggar, Shewanella oneidensis mr-1 nanowires are outer membrane and periplasmic extensions of the extracellular electron transport components, Proc. Natl. Acad. Sci. 111 (2014) 12883-12888.

[4] S. E. Barchinger, S. Pirbadian, C. Sambles, C. S. Baker, K. M. Leung, N. J. Burroughs, M. Y. ElNaggar, J. H. Golbeck, Regulation of gene expression in shewanella oneidensis mr-1 during electron acceptor limitation and bacterial nanowire formation, Appl. Environ. Microbiol. 82 (2016) 5428-5443.

[5] R. Y. Adhikari, N. S. Malvankar, M. T. Tuominen, D. R. Lovley, Conductivity of individual geobacter pili, RSC Adv. 6 (2016) 8354-8357. 
[6] H. Phan, M. D. Yates, N. D. Kirchhofer, G. C. Bazan, L. M. Tender, T.-Q. Nguyen, Biofilm as a redox conductor: a systematic study of the moisture and temperature dependence of its electrical properties, Phys. Chem. Chem. Phys. 18 (2016) 17815.

[7] N. L. Ing, T. D. Nusca, A. I. Hochbaum, Geobacter sulfurreducens pili support ohmic electronic conduction in aqueous solution, Phys. Chem. Chem. Phys. 19 (2017) 21791.

[8] P. Subramanian, S. Pirbadian, M. Y. El-Naggar, G. J. Jensen, Ultrastructure of shewanella oneidensis mr-1 nanowires revealed by electron cryotomography, Proc. Nat. Acad. Sci. USA 115 (2018) E3246E3255.

[9] K. M. Leung, G. Wanger, M. Y. El-Naggar, Y. A. Gorby, G. Southam, W. M. Lau, J. Yang, Shewanella oneidensis mr-1 bacterial nanowires exhibit p-type, tunable electronic behavior, ACS Nano 13 (2013) $2407-2411$.

[10] T. Clarke, M. Edwards, A. Gates, A. Hall, G. White, J. Bradley, C. Reardon, L. Shi, A. Beliaev, M. Marshall, Z. Wang, N. Watmough, J. Fredrickson, J. Zachara, J. Butt, D. Richardson, Structure of a bacterial cell surface decaheme electron conduit, Proc. Natl. Acad. Sci. USA 108 (2011) 9384-9389.

[11] M. J. Edwards, A. Hall, L. Shi, J. K. Fredrickson, J. M. Zachara, J. N. Butt, D. J. Richardson, T. A. Clarke, The crystal structure of the extracellular 11-heme cytochrome unda reveals a conserved 10-heme motif and defined binding site for soluble iron chelates, Structure 20 (2012) 1275-1284.

[12] M. J. Edwards, N. A. Baiden, A. Johs, S. J. Tomanicek, L. Liang, L. Shi, J. K. Fredrickson, J. M. Zachara, A. J. Gates, J. N. Butt, D. J. Richardson, T. A. Clarke, The x-ray crystal structure of shewanella oneidensis omca reveals new insight at the microbemineral interface, FEBS Letters 588 (2014) 1886-1890.

[13] M. J. Edwards, G. F. White, M. Norman, A. Tome-Fernandez, E. Ainsworth, L. Shi, J. K. Fredrickson, J. M. Zachara, J. N. Butt, D. J. Richardson, T. A. Clarke, Redox linked flavin sites in extracellular decaheme proteins involved in microbe-mineral electron transfer, Sci. Rep. 5 (2015) 11677.

[14] R. S. Hartshorne, C. L. Reardon, D. Ross, J. Nuester, T. A. Clarke, A. J. Gates, P. C. Mills, J. K. Fredrickson, J. M. Zachara, L. Shi, A. S. Beliaev, M. J. Marshall, M. Tien, S. Brantley, J. N. Butt, D. J. Richardson, Characterization of an extracellular conduit between bacteria and the extracellular environment, Proc. Natl. Acad. Sci. USA 106 (2009) 22169-22174.

[15] G. F. White, Z. Shi, L. Shi, Z. Wang, A. C. Dohnalkova, M. J. Marshall, J. K. Fredrickson, J. M. Zachara, J. N. Butt, D. J. Richardson, T. A. Clarke, Rapid electron exchange between surface-exposed bacterial cytochromes and fe(iii) minerals, Proc. Nat. Acad. Sci. USA 110 (2013) 6346-6351.

[16] H. Yi, K. P. Nevin, B.-C. Kim, A. E. Franks, A. Klimes, L. M. Tender, D. R. Lovley, Selection of a variant of geobacter sulfurreducens with enhanced capacity for current production in microbial fuel cells, Biosens. Bioelect. 24 (2009) 3498-3503.

[17] L. A. Fitzgerald, E. R. Petersen, B. J. Gross, C. M. Soto, B. R. Ringeisen, M. Y. El-Naggar, J. C. Biffinger, Aggrandizing power output from shewanella oneidensis mr-1 microbial fuel cells using calcium chloride, Biosens. Bioelect. 31 (2012) 492-498.

[18] D. E. Ross, J. M. Flynn, D. B. Baron, J. A. Gralnick, D. R. Bond, Towards electrosynthesis in shewanella: energetics of reversing the mtr pathway for reductive metabolism, PLosS ONE 6 (2011) e16649.

[19] D. R. Lovley, Electromicrobiology, Annu. Rev. Microbiol. 66 (2012) 391-409.

[20] U. Schroder, F. Harnisch, L. T. Angenent, Microbial electrochemistry and technology: terminology and classification, Energy Environ. Sci. 8 (2015) 513-519. 
[21] K. Zhuang, E. Ma, D. R. Lovley, R. Mahadevan, The design of long-term effective uranium bioremediation strategy using a community metabolic model, Biotechnol Bioeng. 109 (2012) 2475-2483.

[22] O. Kokhan, N. S. Ponomarenko, P. R. Pokkuluri, M. Schiffer, K. L. Mulfort, D. M. Tiede, Bidirectional photoinduced electron transfer in ruthenium(ii)-tris- bipyridyl-modified ppca, a multi-heme ctype cytochrome from geobacter sulfurreducens, J. Phys. Chem. B 119 (2015) 7612-7624.

[23] N. S. Wigginton, K. M. Rosso, B. H. Lower, L. Shi, M. F. J. Hochella, Electron tunneling properties of outer-membrane decaheme cytochromes from shewanella oneidensis, Geochim. Cosmochim. Acta 71 (2007) 543-555.

[24] H. S. Byun, S. Pirbadian, A. Nakano, L. Shi, M. Y. El-Naggar, Kinetic monte carlo simulations and molecular conductance measurements of the bacterial decaheme cytochrome mtrf, ChemElectroChem 1 (2014) 1932-1939.

[25] M. Breuer, P. Zarzycki, J. Blumberger, K. M. Rosso, Thermodynamics of electron flow in the bacterial deca-heme cytochrome mtrf, J. Am. Chem. Soc. 134 (2012) 9868-9871.

[26] M. Breuer, P. Zarzycki, L. Shi, T. A. Clarke, M. Edwards, J. Butt, D. J. Richardson, J. K. Fredrickson, J. M. Zachara, J. Blumberger, K. M. Rosso, Molecular structure and free energy landscape for electron transport in the deca-heme cytochrome mtrf, Biochem. Soc. Trans. 40 (2012) 1198-1203.

[27] M. Breuer, K. M. Rosso, J. Blumberger, Electron flow in multi-heme bacterial cytochromes is a balancing act between heme electronic interaction and redox potentials, Proc. Nat. Acad. Sci. USA 111 (2014) 611-616.

[28] J. Blumberger, Recent advances in the theory and molecular simulation of biological electron transfer reactions, Chem. Rev. 115 (2015) 11191-11238.

[29] X. Jiang, Z. Futera, M. E. Ali, F. Gajdos, G. F. v. Rudorff, A. Carof, M. Breuer, J. Blumberger, Cysteine linkages accelerate electron flow through tetra-heme protein stc, J. Am. Chem. Soc. 139 (2017) 17237-17240.

[30] N. S. Wigginton, K. M. Rosso, M. F. Hochella, Mechanisms of electron transfer in two decaheme cytochromes from a metal-reducing bacterium, J. Phys. Chem. B 111 (2007) 12857-12864.

[31] A. Barrozo, M. Y. El-Naggar, A. I. Krylov, Distinct electron conductance regimes in bacterial decaheme cytochromes, Angew. Chem. Int. Ed. 57 (2018) 6805-6809.

[32] C. D. Bostick, S. Mukhopadhyay, I. Pecht, M. Sheves, D. Cahen, D. D. Lederman, Protein bioelectronics: A review of what we do and do not know, Reports Prog. Phys. 81 (2018) 26601.

[33] N. Amdursky, D. Marchak, L. Sepunaru, I. Pecht, M. Sheves, D. Cahen, Electronic transport via proteins, Adv. Mater. 26 (2014) 7142-7161.

[34] M. Breuer, K. M. Rosso, J. Blumberger, J. N. Butt, Multi-heme cytochromes in shewanella oneidensis mr-1: Structures, functions and opportuntities, J. R. Soc. Interface 12 (2015) 20141117.

[35] M. Y. El-Naggar, G. Wanger, K. Leung, T. D. Yuzvinsky, G. Southam, J. Yang, W. M. Lau, K. H. Nealson, Y. A. Gorby, Electrical transport along bacterial nanowires from shewanella oneidensis mr-1, Proc. Natl. Acad. Sci. USA 107 (2010) 18127-18131.

[36] M. D. Yates, J. P. Golden, J. Roy, S. M. Strycharz-Glaven, S. Tsoi, J. S. Erickson, M. Y. El-Naggar, S. C. Bartone, L. M. Tender, Thermally activated long range electron transport in living biofilms, Phys. Chem. Chem. Phys. 17 (2015) 32564. 
[37] M. D. Yates, B. J. Eddie, N. J. Kotloski, N. Lebedev, A. P. Malanoski, B. Lin, S. M. Strycharz-Glaven, L. M. Tender, Toward understanding long-distance extracellular electron transport in an electroautotrophic microbial community, Energy Environ. Sci. 9 (2016) 3544.

[38] N. S. Malvankar, M. Vargas, K. P. Nevin, A. E. Franks, C. Leang, B.-C. Kim, K. Inoue, T. Mester, S. F. Covalla, J. P. Johnson, V. M. Rotello, M. T. Tuominen, D. R. Lovley, Tunable metallic-like conductivity in microbial nanowire networks, Nature Nanotech. 6 (2011) 573.

[39] N. Lebedev, S. Mahmud, I. Griva, A. Blom, L. M. Tender, On the electron transfer through geobacter sulfurreducens pila protein, J. Polym. Sci., Part B: Polym. Phys.

[40] D. A. Boyd, R. M. Snider, J. S. Erickson, J. N. Roy, S. M. Strycharz-Glaven, L. M. Tender, Theory of redox conduction and the measurement of electron transport rates through electrochemically active biofilms, John Wiley \& Sons, Inc., 2015, book section 6, pp. 177-209.

[41] M. L. Rodrigues, T. F. Oliveira, I. A. C. Pereira, M. Archer, X-ray structure of the membrane-bound cytochrome c quinol dehydrogenase nrfh reveals novel haem coordination, Embo J. 25 (2006) 5951.

[42] V. B. Paixao, C. A. Salgueiro, L. Brennan, G. A. Reid, S. K. Chapman, D. L. Turner, The solution structure of a tetraheme cytochrome from shewanella frigidimarina reveals a novel family structural motif, Biochemistry 47 (2008) 11973-11980.

[43] P. Taylor, S. L. Pealing, G. A. Reid, S. K. Chapman, M. D. Walkinshaw, Structural and mechanistic mapping of a unique fumarate reductase, Nat. Struct. Mol. Biol. 6 (1999) 1108.

[44] M. Youngblut, E. T. Judd, V. Srajer, B. Sayyed, T. Goelzer, S. J. Elliott, M. Schmidt, A. A. Pacheco, Laue crystal structure of shewanella oneidensis cytochrome c nitrite reductase from a high-yield expression system., J. Biol. Inorg. Chem. 17 (2012) 647-662.

[45] C. G. Mowat, E. Rothery, C. S. Miles, L. McIver, M. K. Doherty, K. Drewette, P. Taylor, M. D. Walkinshaw, S. K. Chapman, G. A. Reid, Octaheme tetrathionate reductase is a respiratory enzyme with novel heme ligation, Nat. Struct. Mol. Biol. 2004 (2004) 1023-1024.

[46] T. A. Clarke, M. Edwards, A. Gates, A. Hall, G. White, J. Bradley, C. Reardon, L. Shi, A. Beliaev, M. J. Marshall, et al. structure of a bacterial cell surface decaheme electron conduit, Proc. Natl. Acad. Sci. USA 108 (2011) 9384-9389.

[47] M. J. Edwards, G. F. White, M. Norman, A. Tome-Fernandez, E. Ainsworth, L. Shi, J. K. Fredrickson, J. M. Zachara, J. N. Butt, D. J. Richardson, et al. redox linked flavin sites in extracellular decaheme proteins involved in microbe-mineral electron transfer, Sci. Rep. 5 (2015) 11677.

[48] Y. Liu, Z. Wang, J. Liu, C. Levar, M. J. Edwards, J. T. Babauta, D. W. Kennedy, Z. Shi, H. Beyenal, D. R. Bond, T. A. Clarke, J. N. Butt, D. J. Richardson, K. M. Rosso, J. M. Zachara, J. K. Fredrickson, L. Shi, A trans-outer membrane porin-cytochrome protein complex for extracellular electron transfer by geobacter sulfurreducens pca, Environ. Microbiol. Rep. 6 (2014) 776-785.

[49] N. F. Polizzi, S. S. Skourtis, D. N. Beratan, Physical constraints on charge transport through bacterial nanowires, Faraday Discuss. 155 (2012) 43-62.

[50] Y. Zhang, C. Liu, A. Balaeff, S. S. Skourtis, D. N. Beratan, Biological charge transfer via flickering resonance, Proc. Nat. Acad. Sci. USA 111 (2014) 10049-10054.

[51] S. Pirbadian, M. Y. El-Naggar, Multistep hopping and extracellular charge transfer in microbial redox chains, Phys. Chem. Chem. Phys. 14 (2012) 13802. 
[52] J. G. Simmons, Generalized formula for the electric tunnel effect between similar electrodes separated by a thin insulating film, J. Appl. Phys. 34 (1963) 1793-1803.

[53] J. Zhao, J. J. Davis, M. S. P. Sansom, A. Hung, Exploring the electronic and mechanical properties of protein using conducting atomic force microscopy, J. Am. Chem. Soc. 126 (2004) 5601-5609.

[54] L. Sepunaru, I. Pecht, M. Sheves, D. Cahen, Solid-state electron transport across azurin: From a temperature-independent to a temperature activated mechanism, J. Am. Chem. Soc. 133 (2011) 24212423.

[55] J. A. Fereiro, X. Yua, I. Pecht, M. Sheves, J. C. Cuevas, D. Cahen, Tunneling explains efficient electron transport via protein junctions, Proc. Nat. Acad. Sci. USA 115 (2018) E4577-E4583.

[56] N. Amdursky, D. Ferbera, C. A. Bortolotti, D. A. Dolgikh, R. V. Chertkova, I. Pecht, M. Sheves, D. Cahen, Solid-state electron transport via cytochrome c depends on electronic coupling to electrodes and across the protein, Proc. Nat. Acad. Sci. USA 111 (2014) 5556-5561.

[57] C. Guo, X. Yu, S. Refaely-Abramson, L. Sepunaru, T. Bendikov, I. Pecht, L. Kronik, A. Vilan, M. Sheves, D. Cahen, Tuning electronic transport via hepta-alanine peptides junction by tryptophan doping, Proc. Nat. Acad. Sci. USA 113 (2016) 10785-10790. 
Figure 1: Electron transfer over multiple length scales in the bacterium Shewanella Oneidensis. (A) Electronically conducting, micrometer-long cellular appendages. Adapted with permission from Ref. 3. (B) Model of a cellular appendage as an extension of the outer-membrane and the periplasm (light green). Mtr$\mathrm{CAB}$ complexes comprised of the deca-heme proteins MtrC and MtrA and the non-heme protein MtrA are inserted in the outer membrane and form a connected electron transport path along the appendage. Adapted with permission from Ref. 3. (C) Crystal structure of MtrC (pdb code $4 \mathrm{LM} 8^{13}$ ), with heme cofactors shown in green and Fe atoms in pink. The assumed electron input site, heme 10, is shown at the bottom and the assumed electron egress site, heme 5, at the top. (D) Close-up on the heme 10 - heme 9 pair forming a "stacked motif" with heme edge-to-edge distance of about $4 \AA$. Two orbitals that contribute to electronic coupling between the two hemes are indicated by blue/grey and red/yellow positive/negative isosurfaces. The orbitals belong to the redox active $\mathrm{Fe}^{2+} d\left(\mathrm{t}_{2 \mathrm{~g}}\right)$ manifold of states that mix weakly with orbitals of the heme ring and axial His ligands. Adapted with permission from Ref. 27.

Figure 2: Experiments probing electron transfer (ET) in multi-heme cytochromes. (A) ET through the MtrCAB complex from S. Oneidensis, inserted in a proteoliposome. MtrC, MtrA and MtrB are indicated by a blue sphere, green rectangle and black lines, respectively. Heme cofactors are indicated by red diamonds. Electrons are provided by methylviologen (MV) in the periplasmatic side of the proteoliposome and transferred via MtrCAB to the exterior, on to an iron oxide nanoparticle that is reduced to soluble $\mathrm{Fe}^{2+}$. (B) Time resolved concentration profiles of oxidized MV are shown for different mineral surfaces. The initial concentration of MV and the final concentration of aqueous $\mathrm{Fe}^{2+}$ are compared in the inset. (C) Photo-induced electron injection in the tri-heme MHC PpcA from Geobacter sulfurreducans. Heme cofactors are shown in blue and are annotated with roman numerals. $\mathrm{A} \mathrm{Ru}(\mathrm{II})$ chromophore $\left(\mathrm{L}=(\mathrm{bpy})_{2}, \mathrm{X}=(\mathrm{bpy})\right)$ is covalently linked to amino acids that are mutated to Cys (shown in red). (D) Irradiation with visible light gives rise to $\mathrm{Ru}$ metal-to-ligand charge transfer excitation (MLCT), formation of a (long-lived) triplet state and electron injection to the nearby Fe(III)-heme. Adapted with permission from Ref. 22.

Figure 3: Electron transport (ETp) measurements on multi-heme cytochromes (MHCs). (A) Schematic of a scanning tunneling microscope (STM) probing ETp through a multiheme protein (MtrF). The STM tip is represented by grey spheres and the Au substrate by golden spheres. The protein backbone of MtrF is shown in grey and heme cofactors are indicated in blue. A bias voltage $(V)$ between the tip and the $\mathrm{Au}$ substrate induces current flow $(I)$ through the protein. Adapted with permission from Ref. 24. (B) $I-V$ characteristic for the deca-heme proteins $\mathrm{MtrC},{ }^{23} \mathrm{OmcA}^{23}$ and $(\mathrm{C}) \mathrm{MtrF}^{24}$ from STM measurements on $\mathrm{Au}(111)$ using a similar configuration as shown in panel (A). While the curve for OmcA fits the coherent elastic tunneling model $(\mathrm{CET})^{52,53}$ well, the one for MtrC exhibits kinks at positive bias voltage interpreted to arise from participation of localized heme states. For MtrF, measurements are carried out under high and low humidity conditions (blue and red lines, respectively). Fits to a hopping model assuming transport via stepwise heme-to-heme ET are shown in grey. Adapted with permission from Refs. 34 and 24. 
A cellular appendages

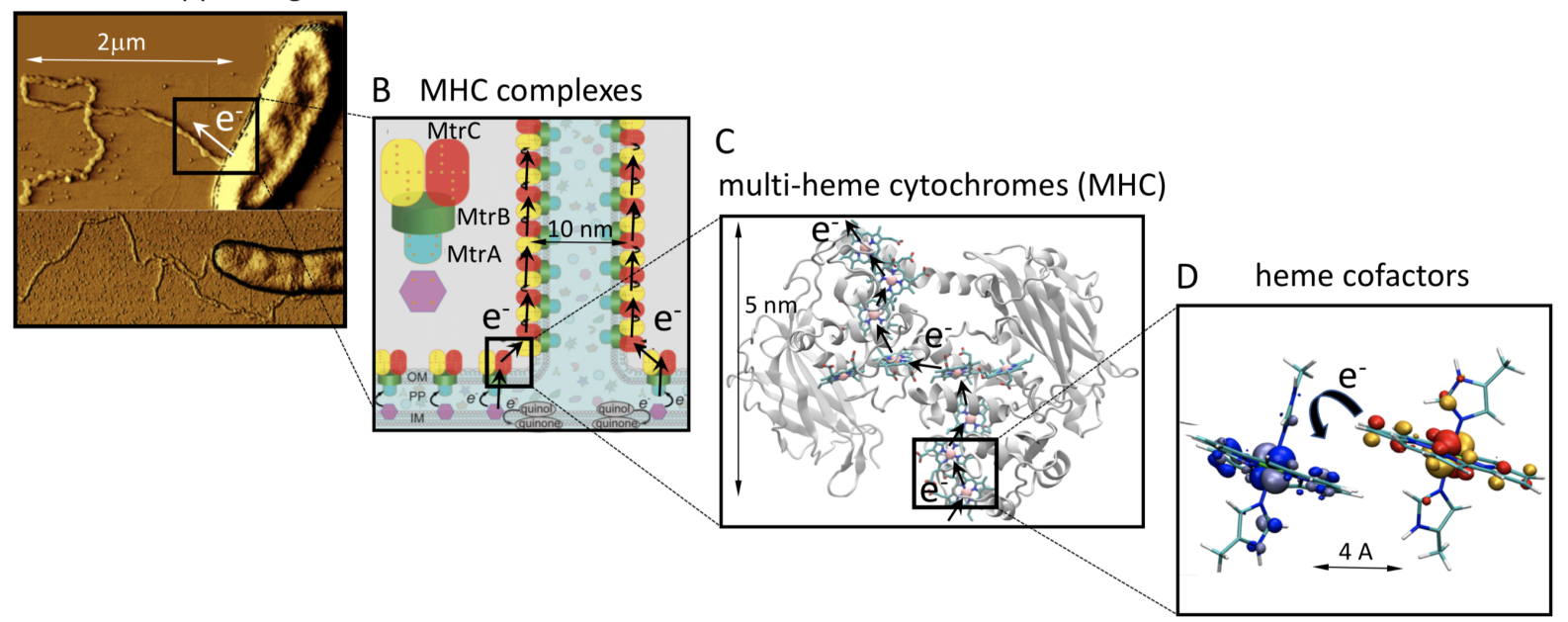

Figure 1. 

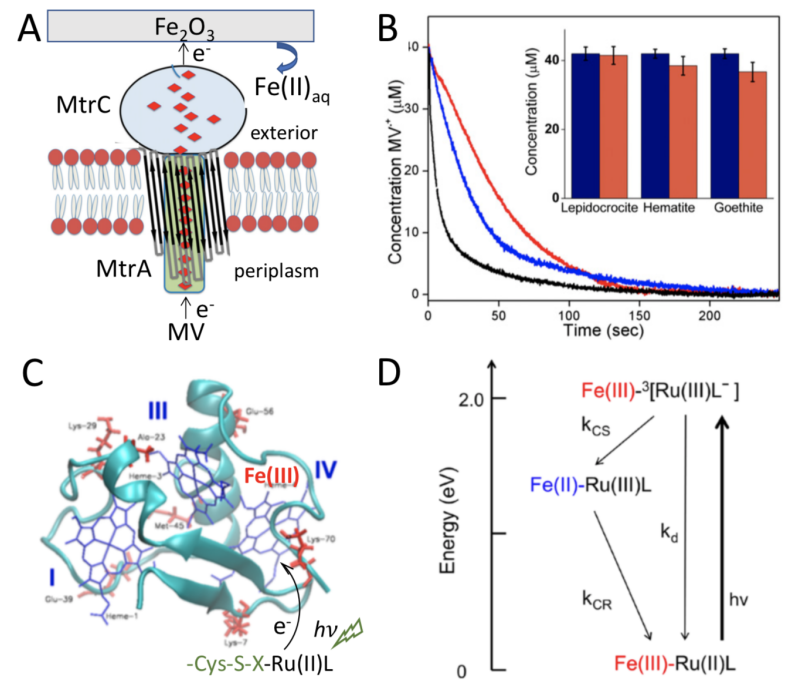

Figure 2 . 

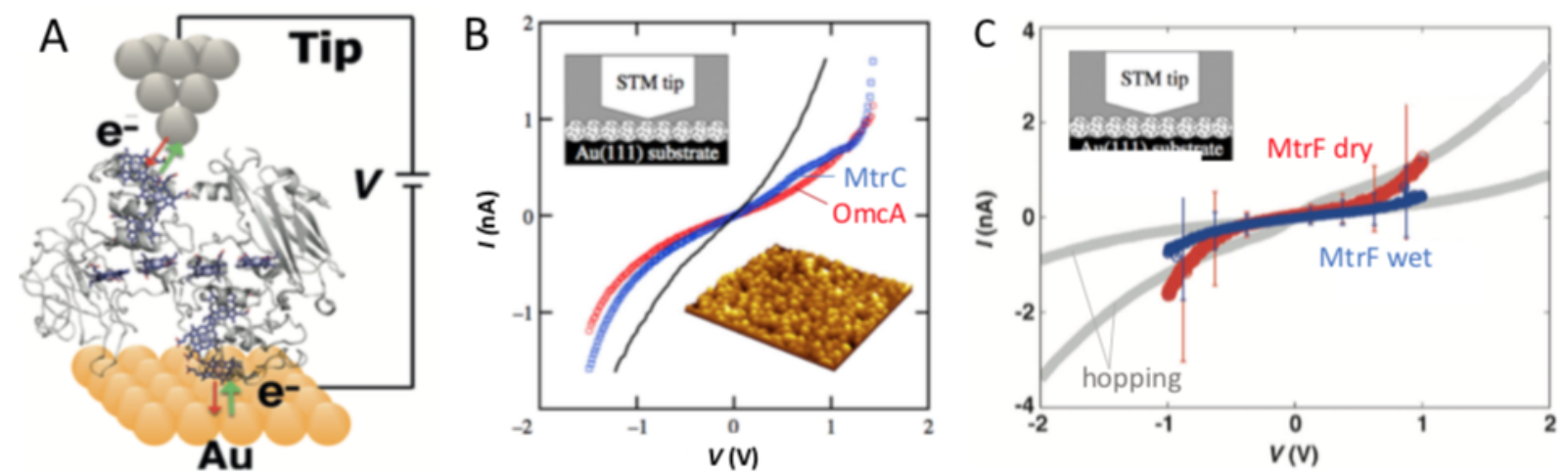

Figure 3. 